\title{
Early stages of phase decomposition in NiAu alloy thin films studied by in situ TEM using ultrafast quenching methods
}

Johanna Schubert $^{1}$, Johannes Will ${ }^{2}$, Thomas Przybilla ${ }^{1}$, Mingjian $\mathrm{Wu}^{1}$ and Erdmann Spiecker ${ }^{1}$

${ }^{1}$ Institute of Micro- and Nanostructure Research (IMN) \& Center for Nanoanalysis and Electron Microscopy (CENEM), Interdisciplinary Center for Nanostructured Films (IZNF), Department of Materials Science and Engineering, Friedrich-Alexander University Erlangen-Nürnberg (FAU), Erlangen, Germany, Erlangen, Bayern, Germany, ${ }^{2}$ Institute of Micro- and Nanostructure Research (IMN) \& Center for Nanoanalysis and Electron Microscopy (CENEM), Interdisciplinary Center for Nanostructured Films (IZNF), Department of Materials Science and Engineering, Friedrich-Alexander University Erlangen-Nürnberg (FAU), Erlangen, Germany, Germany

Discontinuous reactions appear in a great variety of alloys undergoing phase decomposition. Typically the reaction starts at grain boundaries (GBs) where nuclei form in the supersaturated alloy phase. The GB is crucial for the material transport and migrates into one of the grains, leaving behind a lamellar structure of the two decomposed phases [1]. The Ni-Au system exhibits a large lattice misfit of $>10 \%$ associated with a miscibility gap covering the whole concentration range reaching up to $810^{\circ} \mathrm{C}$ at its maximum. It therefore constitutes an ideal model system to gain insight into the influence of lattice strain on the decomposition behavior. Methodologically, the large difference in atomic number is ideally suited for Z-contrast scanning transmission electron microscopy (STEM) which can be combined with analytical techniques like electron energy loss spectroscopy (EELS). For NiAu thin films the alloyed high temperature state can be preserved at room temperature using fast quenching techniques. By subsequent stepwise heating at temperatures inside the miscibility gap the very early stages of phase decomposition can be monitored and investigated in detail.

To achieve this a $\mathrm{Ni}(25 \mathrm{~nm})$ and $\mathrm{Au}(15 \mathrm{~nm})$ bilayer thin film is deposited on the amorphous $\mathrm{SiN}_{\mathrm{x}}$ membrane of a MEMS chip using e-beam physical vapor deposition. This MEMS chip is part of a TEM heating device (DENSsolutions Wildfire) which enables ultrafast cooling with quenching rates exceeding $1000 \mathrm{~K} / \mathrm{s}$ as determined by direct in situ temperature measurements in the TEM [2]. To first form an alloyed film, the bilayer stack is heated above the miscibility gap at $950^{\circ} \mathrm{C}$ for 30 seconds and is subsequently quenched to room temperature, preserving the solid solution state. During this step, initial stages of solid-state dewetting occur and cannot be fully suppressed. However, in large parts of the film the film thickness is retained while considerable grains with [111]-texture form through grain growth [3]. In Fig 1a the importance of the cooling speed is illustrated. Whereas a sample with a slower cooling speed (blue curve) already shows phase decomposition, albeit restricted to dislocations along small angle $\mathrm{GBs}$, the sample quenched below $50^{\circ} \mathrm{C}$ within one second exhibits no detectable phase decomposition. This comparison reveals the absolute necessity of very fast cooling rates as available via the utilized modern MEMS based heating technology to achieve an alloyed reference state at room temperature.

The GBs of the sample already showing contrast modulations were post characterized by Transmission Kikuchi Diffraction (TKD) to determine the relative orientation of the grains. For the grains A and B indicated in Fig. 1c the analysis yields an in-plane rotation of $5.1^{\circ}$ and a small tilt of the [111] out of plane orientation of $0.2^{\circ}$. Neglecting the tilt and assuming perfect dislocations the dislocation spacing is calculated to $2.9 \mathrm{~nm}$ using Frank's rule. This is well in-line with the measured distance between the modulations of $3.2 \mathrm{~nm}$, which would imply an angle of $4.7^{\circ}$. Furthermore, high resolution STEM imaging was performed, confirming that the center of each bright-dark contrast modulation correlates with a dislocation core. In addition EELS reveals $\mathrm{Ni}$ segregation on the compressive side of the dislocation strain field (leading to dark Z contrast in STEM), whereas the larger $\mathrm{Au}$ atoms segregate on the tensile side (bright $\mathrm{Z}$ contrast), as expected to reduce the local strain. Overall, this analysis reveals the importance of GBs and dislocations including their local strain fields in the first stages of phase decomposition in high misfit alloys like NiAu. 
To enable a detailed chemical and structural characterization of the decomposition process, the chip-based TEM heating system was utilized for sequential in situ annealing in steps of a few seconds interrupted by fast quenching to room temperature for TEM analysis. The image series in Fig 2 was generated in this way for an annealing temperature of $550^{\circ} \mathrm{C}$ and contains two GB triple points acting as nucleation sites for discontinuous phase decomposition. We compared the interrupted heating studies with continuous in situ heating experiments at same temperature and found identical decomposition phenomena, showing that the intermediate quenching has almost no influence on the reaction. The phase decomposition in the NiAu thin films starts preferably at triple points with a Ni nucleus forming in one grain (e.g. grain A at triple point I) and subsequently bulging into one of the adjacent grains (here B). TKD reveals a much larger misorientation between grains A and B compared to grains $\mathrm{A}$ and $\mathrm{C}$, suggesting that the reaction preferentially proceeds at the energetically less favorable GB. At triple point II phase decomposition is accompanied by GB migration including migration of the triple point itself. The Ni-rich nucleus forms in grain $\mathrm{D}$ in the vicinity of the triple point II. As the reaction front moves into grain B an alternating Au- and Ni-rich lamellar structure evolves from grains C, D and E. Each lamella maintains the orientation of its native grain, i.e. lamellae 2, 3 and 4 exhibit the orientation of grains E, D and C, respectively. The new interface that is created between the lamellae is thus an extension of the low energy GBs between grains C and D, and D and E, respectively. Similar for triple point I, the reaction front evolves along the highest energy GB. Thus triple points act as nucleation sites and can be further included in the reaction front.

Overall, we demonstrate an elegant approach utilizing a MEMS based TEM heating system to monitor early stages of phase decomposition in NiAu films used as model alloy system with large misfit and miscibility gap. Besides methodological developments, the important role of GBs, triple points, dislocations and their strain fields on early stages of phase decomposition are presented and will be discussed in the light of existing models [4].
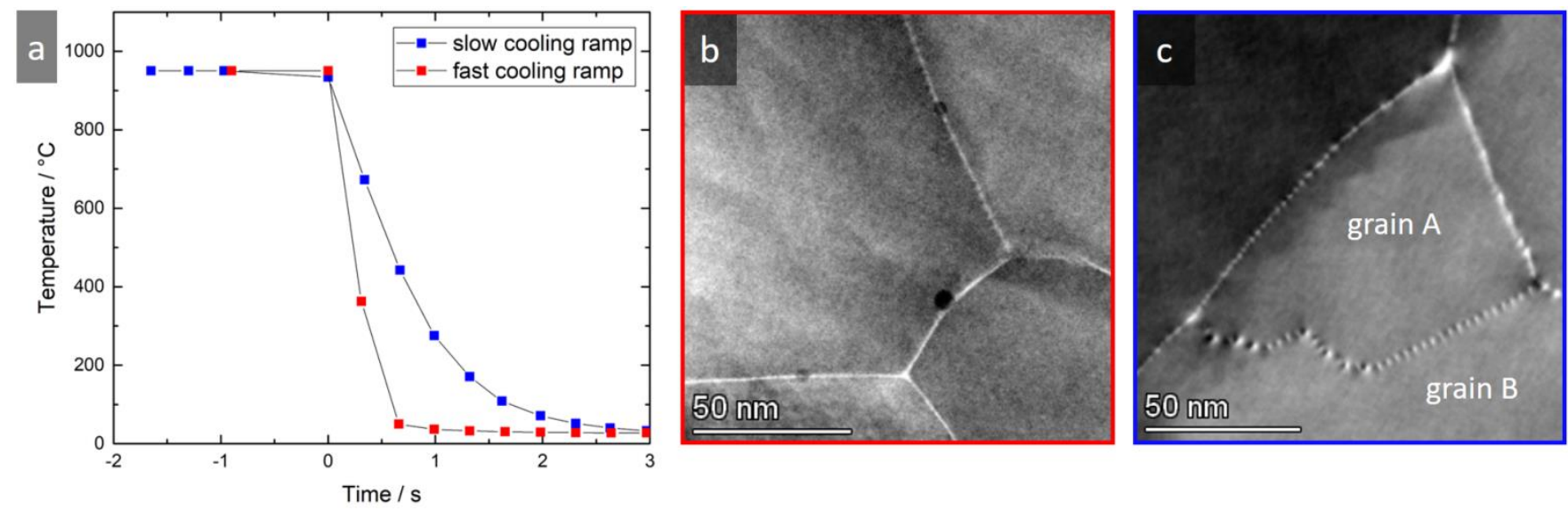

Figure 1. a) Cooling ramps of two samples with different cooling rates after annealing the $\mathrm{NiAu}$ film at $950^{\circ} \mathrm{C}$ for $30 \mathrm{~s}, \mathrm{~b}$ ) STEM image of the sample cooled below $50^{\circ} \mathrm{C}$ in under $1 \mathrm{~s}$ (red) and c) of a sample cooled to this point in almost 3 seconds (blue), already exhibiting phase decomposition at single dislocations in a small angle grain boundary. 

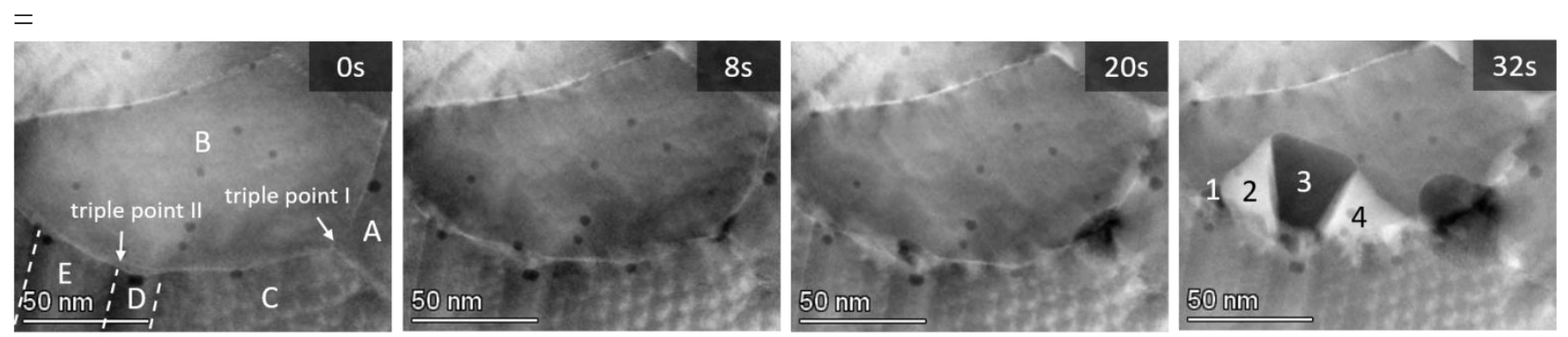

Figure 2. Interrupted in situ annealing at $550^{\circ} \mathrm{C}$ of a $\mathrm{NiAu}$ alloy thin film revealing discontinuous phase decomposition at two neighboring triple points.

\section{References}

[1] Manna, I.; Pabi, S.K.; Gust, W. International Materials Reviews 2001 46/2, 53-91

[2] Niekiel, F.; Kraschewski, S.M.; Müller, J.; Butz, B.; Spiecker, E. Ultramicroscopy 2017 176, 161-169

[3] Niekiel, F.; Kraschewski, S.M.; Schweizer, P.; Butz, B.; Spiecker, E. Acta Materialia 2016 115, 230-241

[4] The authors thank the DFG for financial support within the framework of the research training group GRK1896 'In situ Microscopy with Electrons, X-rays and Scanning Probes’ 